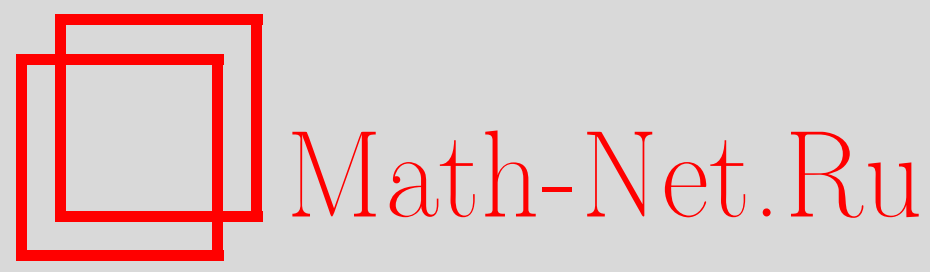

В. А. Таров, Гладко меняющиеся функции и совершенные уточненные порядки, Матем. заметки, 2004, том 76, выпуск 2, 258-264

DOI: https://doi.org/10.4213/mzm104

Использование Общероссийского математического портала Math-Net.Ru подразумевает, что вы прочитали и согласны с пользовательским соглашением http://www . mathnet.ru/rus/agreement

Параметры загрузки:

IP: 54.162 .85 .209

26 апреля 2023 г., $16: 12: 37$

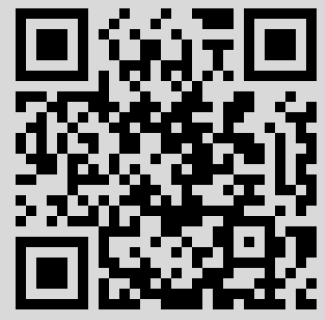




\title{
ГЛАДКО МЕНЯЮЩИЕСЯ ФУНКЦИИ И СОВЕРШЕННЫЕ УТОЧНЕННЫЕ ПОРЯДКИ
}

\author{
B. А. Таров
}

В статье показано, что функция $h(r)$ является гладко меняющейся функцией порядка $\rho$ тогда и только тогда, когда функция $\rho(r)=(\ln h(r)) / \ln r$ является совершенньмм уточненным порядком, т.е. бесконечно дифференцируемой в некоторой окрестности $+\infty$ функцией, для которой выполняются условия $\lim _{r \rightarrow+\infty} \rho(r)=\rho, \rho \in \mathbb{R}$, и $\lim _{r \rightarrow+\infty} r^{n} \ln r \rho^{(n)}(r)=0$ для всех $n \in \mathbb{N}$. В статье также получены некоторые следствия приведенного выше результата.

Библиография: 4 названия.

1. Введение и формулировка результатов. При изучении асимптотических свойств функций различных классов часто имеют дело с положительньгми в окрестности $+\infty$ функциями. Например, для целой не равной тождественно константе функции $f(z), z \in \mathbb{C}$, положительной в некоторой окрестности $+\infty$ является (см. [1, гл. 1 , $\S 1])$ функция $\ln M(r, f)$, где $M(r, f)=\max \{|f(z)|:|z|=r\}$. Функция $n(r, f)$ - число нулей $f(z)$ в круге $\{z:|z| \leqslant r\}$ - также положительна в некоторой окрестности $+\infty$, если $f(z)$ обрашается в нуль хотя бы в одной точке.

В многочисленных работах, особенно в работах, касающихся роста субгармонических функций (частным случаем которых являются логарифмы модулей целых функций) положительные в окрестности $+\infty$ функции сравниваются с функциями вида $h(r)=r^{\rho(r)}$, где $\rho(r)-$ уточненньй порядок.

ОпредЕЛЕниЕ 1. Вещественнозначная дифференцируемая на некотором луче ( $a$, $+\infty), a \geqslant 0$, функция $\rho(r)$ назьвается уточненным поряджом, если

$$
\lim _{r \rightarrow+\infty} \rho(r)=\rho, \quad \rho \in \mathbb{R}, \quad \lim _{r \rightarrow+\infty}(r \ln r) \rho^{\prime}(r)=0 .
$$

Функция $h(r)=r^{\rho(r)}$, где $\rho(r)$ - уточненньй порядок, является, как это следует из леммы $5[1$, гл. $1, \S 12]$, правильно меняющейся функцией порядка $\rho$.

Работа выполнена при финансовой поддержке Российского фонда фундаментальных исследований, грант № 02-01-01098, и программы “Ведущие научные школы”, грант № 00-15-96189. 
ОпРЕДЕЛЕНИЕ 2. Измеримая положительная на некотором луче $(a,+\infty), a \geqslant 0$, функция $h(r)$ называется правильно меняющейся порядка $\rho, \rho \in \mathbb{R}$, если для любого положительного числа $k$

$$
\lim _{r \rightarrow+\infty} \frac{h(k r)}{h(r)}=k^{\rho} .
$$

ОПРЕДЕЛЕНИЕ 3. Правильно меняющаяся функция нулевого порядка назьвается медленно меняющейся функиией.

Известно (см. [2, теорема 1.8.2]), что любая правильно меняющаяся функция $h(r)$ эквивалентна при $r \rightarrow+\infty$ некоторой гладко меняющейся функции.

ОПРЕДЕЛЕНИЕ 4. Положительная бесконечно дифференцируемая на некотором луче $(a,+\infty), a \geqslant 0$, функция $h(r)$ называется гладко меняющейся порядка $\rho, \rho \in \mathbb{R}$, если

$$
\lim _{r \rightarrow+\infty}\left(\ln h\left(e^{r}\right)\right)^{\prime}=\rho, \quad \lim _{r \rightarrow+\infty}\left(\ln h\left(e^{r}\right)\right)^{(n)}=0 \quad \forall n \in \mathbb{N} \backslash\{1\} .
$$

Сформулируем в виде теоремы известное (см. [2, п. 1.8.1] и [3, лемма 9]) утверждение.

ТеоремА А. Положительная бесконечно дифференцируемая на некотором луче $(a,+\infty), a \geqslant 0$, функиия $h(r)$ удовлетворяет условиям (1) тогда и только тогда, когда для любого $n \in \mathbb{N}$

$$
\lim _{r \rightarrow+\infty} \frac{r^{n} h^{(n)}(r)}{h(r)}=\rho(\rho-1) \cdots(\rho-n+1) .
$$

ОПРЕДЕЛЕНИЕ 5. Бесконечно дифференцируемьй в некоторой окрестности $+\infty$ уточненньй порядок $\rho(r)$ называется совершенным, если для любого $n \in \mathbb{N}$

$$
\lim _{r \rightarrow+\infty} r^{n} \ln r \rho^{(n)}(r)=0
$$

Основным результатом данной статьи является

ТЕОремА 1. Положительная бесконечно дифференцируемая на некотором луче $(a,+\infty), a \geqslant 0$, функиия $h(r)$ является гладко меняющейся функиией порядка $\rho$ тогда и только тогда, когда функиия

$$
\rho(r)=\frac{\ln h(r)}{\ln r}
$$

является совершенным уточненным порядком, для которого выполняется равенство

$$
\lim _{r \rightarrow+\infty} \rho(r)=\rho .
$$

Для того чтобы сформулировать следующую теорему, нам потребуется еще одно определение. 
ОПРЕДЕЛЕНИЕ 6. Уточненные порядки $\rho_{1}(r)$ и $\rho_{2}(r)$ называются әквивалентны.ми, если

$$
\lim _{r \rightarrow+\infty}\left(\rho_{1}(r)-\rho_{2}(r)\right) \ln r=0
$$

ТЕОРемА 2. Для любого уточненного порядка найдется әквивалентный ему совершенный уточненный порядок.

Отметим, что из теоремы 2 , в частности, следует существование для любого уточненного порядка эквивалентного ему сильного уточненного порядка, т.е. дважды дифференцируемого в окрестности $+\infty$ уточненного порядка, для которого вьполнено (3) при $n=2$.

ТЕОремА 3. Для любой положительной на некотором луче $(a,+\infty), a \geqslant 0$, функиии $\varphi(r)$, удовлетворяющей условию

$$
\left|\varlimsup_{r \rightarrow+\infty} \frac{\ln \varphi(r)}{\ln r}\right|<+\infty
$$

найдутся число $b>$ а и совершенный уточненный порядок $\rho(r)$, такие, что

$$
\begin{aligned}
& \varphi(r) \leqslant r^{\rho(r)} \quad n p u \quad r \geqslant b, \\
& \varlimsup_{r \rightarrow+\infty} \frac{\varphi(r)}{r^{\rho(r)}}=1 .
\end{aligned}
$$

Заметим, вьполнение условия (6) означает, что $\varphi(r)$ имеет конечньй порядок; вьполнение условия $(8)$ - что $\varphi(r)$ имеет при $\rho(r)$ нормальньй (т.е. конечньй положительньй) тип, равный 1 , а $\rho(r)$ является уточненным (в данном случае - совершенным уточненным) порядкком $\varphi(r)$.

Отметим также, что все имеюшиеся вьше определения (кроме определения 5) известны и во многих работах приводились с некоторыми отличиями в формулировках, a в этой статье даны в удобной для рассматриваемых вопросов форме.

2. Доказательство теоремы 1. Везде в доказательстве считаем, что $r \in(a+1$, $+\infty)$.

Необходимость. Пусть $h(r)$ - гладко меняющаяся функция порядка $\rho$. Тогда $h(r)$ является (см. [2, п. 1.8.1]) правильно меняющейся функцией порядка $\rho$ и поэтому представляется в виде

$$
h(r)=r^{\rho} h_{0}(r)
$$

где $h_{0}(r)$ - медленно меняющаяся функция. Из (9) и следует $(5)$, так как известно (см. [4, п. 1.5, свойство $\left.\left.2^{\circ}\right]\right)$, что $\lim _{r \rightarrow+\infty} \ln h_{0}(r) / \ln r=0$.

Из определения 4 вытекает 
УТВЕРЖДЕНИЕ 1. Если $h(r)=r^{\rho} h_{0}(r)$, mo $h(r)$ является гладко меняющейся функиией порядка $\rho$ в том и только том случае, когда $h_{0}(r)$ - гладко меняющаяся функиия порядка 0.

В силу утверждения 1 и теоремы А для любого $n \in \mathbb{N}$

$$
\lim _{r \rightarrow+\infty} \frac{r^{n} h_{0}^{(n)}(r)}{h_{0}(r)}=0 .
$$

Докажем теперь, что для любого $n \in \mathbb{N}$

$$
\lim _{r \rightarrow+\infty} r^{n}\left(\ln h_{0}(r)\right)^{(n)}=0 .
$$

Из (10) при $n=1$ получаем (11) при $n=1$. Предположим, что (11) выполняется для любого $n \in \mathbb{N}$, не превосходящего $k \in \mathbb{N}$. Ввиду равенства

$$
h_{0}^{(k+1)}(r)=\left(h_{0}(r)\left(\ln h_{0}(r)\right)^{\prime}\right)^{(k)}=\sum_{i=0}^{i=k} C_{k}^{i} h_{0}^{(i)}(r)\left(\ln h_{0}(r)\right)^{(k+1-i)}
$$

имеем

$$
\frac{r^{k+1} h_{0}^{(k+1)}(r)}{h_{0}(r)}=r^{k+1}\left(\ln h_{0}(r)\right)^{(k+1)}+\sum_{i=1}^{i=k} C_{k}^{i} \frac{r^{i} h_{0}^{(i)}(r)}{h_{0}(r)} r^{k+1-i}\left(\ln h_{0}(r)\right)^{(k+1-i)} .
$$

Из (12), принимая во внимание вьполнение (10) для любого $n \in \mathbb{N}$ и сделанное предположение, получаем (11) при $n=k+1$. Таким образом, $(11)$ выполняется для любого $n \in \mathbb{N}$ вследствие принципа математической индукции.

Введем функцию

$$
\rho_{0}(r)=\rho(r)-\rho .
$$

Докажем, что для любого $n \in \mathbb{N}$

$$
\lim _{r \rightarrow+\infty} r^{n} \ln r \rho_{0}^{(n)}(r)=0
$$

Из (5) и (13) следует, что

$$
\lim _{r \rightarrow+\infty} \rho_{0}(r)=0
$$

Из (4), (9) и (13) получаем равенство

$$
\rho_{0}(r)=\frac{\ln h_{0}(r)}{\ln r}
$$

Учитьвая (16), находим

$$
r\left(\ln h_{0}(r)\right)^{\prime}=r\left(\ln r \rho_{0}(r)\right)^{\prime}=\rho_{0}(r)+r \ln r \rho_{0}^{\prime}(r) .
$$

Из (17), если принять во внимание (11) при $n=1$ и (15), вытекает (14) при $n=1$. 
Предположим, что (14) вьполняется для любого $n \in \mathbb{N}$, не превосходящего $k \in \mathbb{N}$. Тогда для этих же значений $n$ будет вьполняться и равенство

$$
\lim _{r \rightarrow+\infty} r^{n} \rho_{0}^{(n)}(r)=0
$$

Используя (16), имеем

$$
\left(\ln h_{0}(r)\right)^{(k+1)}=\left(\ln r \rho_{0}(r)\right)^{(k+1)}=\sum_{i=0}^{i=k+1} C_{k+1}^{i}(\ln r)^{(i)} \rho_{0}^{(k+1-i)}(r) .
$$

Из этого равенства вытекает, что

$$
r^{k+1}\left(\ln h_{0}(r)\right)^{(k+1)}=r^{k+1} \ln r \rho_{0}^{(k+1)}(r)+\sum_{i=1}^{i=k+1} C_{k+1}^{i}(-1)^{i+1}(i-1) ! r^{k+1-i} \rho_{0}^{(k+1-i)}(r),
$$

откуда в силу (11) при $n=k+1,(15)$ и (18) для $n$, не превосходящих $k$, следует (14) при $n=k+1$. На основании принципа математической индукции (14) верно для любого $n \in \mathbb{N}$, но тогда и $(3)$ верно для любого $n \in \mathbb{N}$, так как ввиду $(13) \rho^{(n)}(r)=\rho_{0}^{(n)}(r)$ при любом $n \in \mathbb{N}$. Необходимость доказана.

Достаточность. Пусть $\rho(r)=(\ln h(r)) / \ln r$ - совершенный уточненньй порядок, для которого вьполняется (5). Из (3) и (13) следует (14), а значит, и (18), для любого $n \in \mathbb{N}$. Из (5) и (13) получаем (15).

Из (14) при $n=1,(15)$ и (17) находим (11) при $n=1$. Для $n>1$ (11) следует из (19), если учесть (15), а также вьполнение (14) и (18) при любом $n \in \mathbb{N}$.

Условие (10) при $n=1$ вытекает из (11) при $n=1$. Предположим, что (10) вьполняется для любого $n \in \mathbb{N}$, не превосходящего $k \in \mathbb{N}$. Из (12), принимая во внимание вьполнение (11) при любом $n \in \mathbb{N}$ и сделанное предположение, получаем (10) при $n=k+1$. Тогда по принципу математической индукции (10) верно для любого $n \in \mathbb{N}$ и в силу теоремы A $h_{0}(r)$ - гладко меняющаяся функция порядка 0. Ввиду утверждения $1 h(r)$ является гладко меняющейся функцией порядка $\rho$. Достаточность доказана.

Покажем, что используя соотношения, полученные при доказательстве теоремы 1 , можно дать доказательство теоремы А, отличное от доказательства, приведенного в лемме 9 [3].

3. Доказательство теоремы А. Докажем сначала

УТВЕРЖДЕНИЕ 2. Если $h(r)=r^{\rho} h_{0}(r)$, то (2) выполняется при любом $n \in \mathbb{N}$ в том и только том случае, когда (10) выполняется при любом $n \in \mathbb{N}$.

ДокАЗАТЕЛЬСТво. Необходимость. Ввиду (9) имеем

$$
h^{\prime}(r)=\rho r^{\rho-1} h_{0}(r)+r^{\rho} h_{0}^{\prime}(r) .
$$

Из (20), учитывая (2) при $n=1$ и (9), находим, что (10) верно при $n=1$. Предположим, что $(10)$ выполняется для любого $n \in \mathbb{N}$, не превосходящего $k \in \mathbb{N}$. Из (9) и равенства

$$
\left(r^{\rho} h_{0}(r)\right)^{(k+1)}=\sum_{i=0}^{i=k+1} C_{k+1}^{i}\left(r^{\rho}\right)^{(i)} h_{0}^{(k+1-i)}(r)
$$


вытекает, что

$$
\begin{aligned}
\frac{r^{k+1} h^{(k+1)}(r)}{h(r)}= & \frac{r^{k+1} h_{0}^{(k+1)}(r)}{h_{0}(r)}+\sum_{i=1}^{i=k} C_{k+1}^{i} \rho(\rho-1) \cdots(\rho-i+1) \frac{r^{k+1-i} h_{0}^{(k+1-i)}(r)}{h_{0}(r)} \\
& +\rho(\rho-1) \cdots(\rho-k),
\end{aligned}
$$

откуда в силу сделанного предположения и (2) при $n=k+1$ следует (10) при $n=k+1$. На основании принципа математической индукции (10) верно для любого $n \in \mathbb{N}$.

Достаточность. Из (20), учитьвая (9) и (10) при $n=1$, получаем (2) при $n=1$. При $n>1$ (2) следует из (21), так как (10) верно при любом $n \in \mathbb{N}$.

Утверждение 2 доказано.

Из доказательства теоремы 1 вытекает, что (10) верно для любого $n \in \mathbb{N}$ тогда и только тогда, когда для любого $n \in \mathbb{N}$ верно (11).

Осталось доказать следующее

УТВЕРЖДЕНИЕ 3. Для того чтобы для любого $n \in \mathbb{N}$ выполнялось (11), необходимо и достаточно, чтобы для любого $n \in \mathbb{N}$

$$
\lim _{r \rightarrow+\infty}\left(\ln h_{0}\left(e^{r}\right)\right)^{(n)}=0
$$

ДокАЗАТЕЛЬСтво. Необходимость сразу же следует из верного для любого $n \in \mathbb{N}$ равенства

$$
\left(\ln h_{0}\left(e^{r}\right)\right)^{(n)}=\left.\sum_{j=1}^{j=n} b_{j n} e^{j r}\left(\ln h_{0}(t)\right)^{(j)}\right|_{t=\exp r}
$$

где $b_{j n}-$ константы.

Достаточность. При $n=1$ (11) вытекает из (22) при $n=1$. Предположим, что (11) выполняется для любого $n \in \mathbb{N}$, не превосходящего $k \in \mathbb{N}$. Тогда из (23) при $n=k+1$, учитьвая (22) при $n=k+1$ и сделанное предположение, находим, что (11) вьполняется при $n=k+1$. Вследствие принципа математической индукции (11) верно для любого $n \in \mathbb{N}$. Утверждение 3 доказано.

Ввиду утверждения 1 доказана и теорема А.

4. Доказательство теоремы 2. Если $\rho(r)$ - уточненньй порядок, то, как отмечалось во введении, $h(r)=r^{\rho(r)}-$ правильно меняющаяся функция. По теореме 1.8.2 [2] для $h(r)$ найдется эквивалентная ей при $r \rightarrow+\infty$ гладко меняющаяся функция $h_{1}(r)$. Ввиду теоремы $1 \rho_{1}(r)=\left(\ln h_{1}(r)\right) / \ln r-$ совершенньй уточненньй порядок. Из

$$
\lim _{r \rightarrow+\infty} \frac{r^{\rho(r)}}{r^{\rho_{1}(r)}}=1
$$

следует

$$
\lim _{r \rightarrow+\infty}\left(\rho(r)-\rho_{1}(r)\right) \ln r=0
$$

Теорема доказана. 
5. Доказательство теоремы 3. Ввиду (6) найдется число $b_{1}>a+1$ такое, что функция $\varphi(r)$ ограничена на отрезке $\left[b_{1}, b_{2}\right]$ при любом $b_{2} \in\left(b_{1},+\infty\right)$. Тогда из доказательства теоремы 16 [1, гл. $1, \S 12]$ вытекает существование такого уточненного порядка $\rho_{1}(r)$, что

$$
\varphi(r) \leqslant r^{\rho_{1}(r)} \text { при } r \geqslant b_{1} \quad \text { и } \quad \varlimsup_{r \rightarrow+\infty} \frac{\varphi(r)}{r^{\rho_{1}(r)}}=1 .
$$

Вследствие теоремы 1.8.2 [2] для правильно меняющейся функции $r^{\rho_{1}(r)}$ найдутся число $b \geqslant b_{1}$ и гладко меняющаяся функция $h(r)$ такие, что

$$
r^{\rho_{1}(r)} \leqslant h(r) \text { при } r \geqslant b \quad \text { и } \quad \lim _{r \rightarrow+\infty} \frac{r^{\rho_{1}(r)}}{h(r)}=1 .
$$

В силу теоремы $1 \rho(r)=(\ln h(r)) / \ln r$ - совершенньй уточненный порядок. Поэтому из (24) и (25) следует (7) и (8). Теорема доказана.

В заключение выражаю благодарность моему научному руководителю чл.-корр. РАН, профессору В.В. Напалкову за полезное обсуждение статьи.

\section{СПИСОК ЦИТИРОВАННОЙ ЛИТЕРАТУРЫ}

[1] Левин Б. Я. Распределение корней целых функций. М.: ГИТТЛ, 1956.

[2] Bingham N. H., Goldie C. M., Teugels J. L. Regular Variation. Encyclopedia of Mathematics and its Applications. V. 27. Cambridge: Cambridge University Press, 1987.

[3] Balkema A. A., Geluk J. L., De Haan L. An extension of Karamata's Tauberian theorem and its connection with complementary convex functions // Quart. J. Math. Oxford (2). 1979. V. 30. № 120. P. 385-416.

[4] Сенета Е. Правильно меняющиеся функции. М.: Наука, 1985.

Институт математики с ВЦ Уфимского научного центра РАН 